\title{
Three-Dimensional Propagation Analysis of Coupling Efficiency Using Segmentation and Local Normal Mode Expansion for Vertically Coupled Microring Resonator Filter
}

\author{
Tomoyuki Kato and Yasuo Kokubun, Member, IEEE, Member, OSA
}

\begin{abstract}
A novel three-dimensional (3-D) propagation analysis of coupled waveguides using local normal modes and the segmentation along the propagation axis was developed. The coupling efficiency of vertically coupled microring resonators was optimally designed using this analysis. The computation time is much faster and the required memory capacity is much smaller than those of the 3-D finite-difference time-domain (FDTD) method.
\end{abstract}

Index Terms-Coupled mode theory, coupling efficiency, microring resonator, segmentation, three-dimensional (3-D) propagation analysis.

\section{INTRODUCTION}

$\mathbf{M}$ ICRORING resonator add-drop filters [1]-[3] have many attractive features such as functionality, filter response synthesis compactness, and the possibility of dense integration. For higher performance, the vertically coupled microring resonator filter [4]-[6] was proposed and demonstrated. This novel filter has many advantages over the nextgeneration photonic network such as controllability of the coupling efficiency between the busline and the resonator (achieved by controlling the thickness of the separation layer) and the possibility of dense integration resulting from the crossgrid configuration. In this filter, the coupling efficiency between the busline waveguide and the ring resonator is the essential parameter [7] that determines filter response characteristics. Since the busline waveguide and the ring resonator are placed in different layers as shown in Fig. 1, a three-dimensional (3-D) numerical simulation is needed to calculate the coupling efficiency in detail. However, since the upper ring resonator is a bent waveguide in the coupling region, a large 3-D space must be taken as the analysis space. Therefore, the 3-D finitedifference time-domain (FDTD) simulation requires a lot of

Manuscript received October 1, 2004; revised March 28, 2005. This work was supported in part by the Grant-in-Aid for Scientific Research on Priority Areas No. 13026210 from the Ministry of Education, Culture, Sports, Science and Technology, Futaba Electronics Memorial Foundation, and the 21st Century COE Program of the Ministry of Education, Culture, Sports, Science and Technology.

The authors are with the Department of Electrical and Computer Engineering, Graduate School of Engineering, Yokohama National University, Yokohama 240-8501, Japan (e-mail: kokubun@ ynu.ac.jp).

Digital Object Identifier 10.1109/JLT.2005.851320

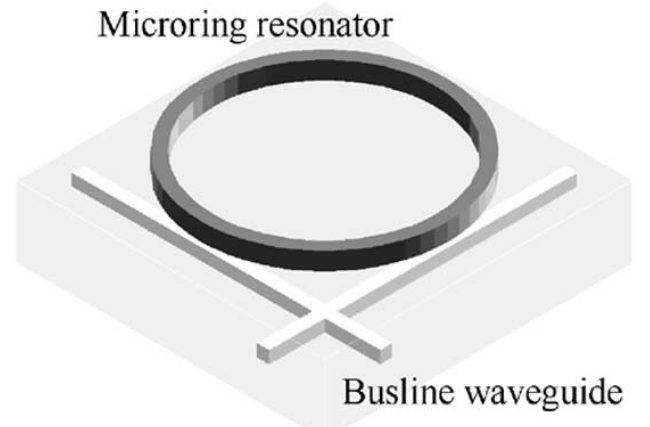

Fig. 1. Vertically coupled microring resonator.

memory and takes a long time, and so it is not suitable for the search of optimum design by varying the parameters.

In this study, a new propagation analysis method to calculate the coupling efficiency of the 3-D bent coupled waveguides is proposed. This method is based on the field expansion of isolated modes in terms of coupled modes of segmented parallel coupled waveguides, which are cascaded to model the bent coupled waveguides. Using this method, the calculation time and memory can be significantly reduced in comparison with those of 3-D FDTD simulation.

\section{THEORY}

Optical beam propagation in a directional coupler is exactly expressed in terms of two eigenmodes (even and odd modes). These eigenmodes are determined by the structure and the arrangement of two waveguides, i.e., the cross-sectional shapes of waveguides, index profile, spacing, and so on. In the case of the coupling between the busline waveguide and the ring resonator waveguide, the spacing between waveguides varies along the propagation direction gradually, and the coupler is usually designed to keep the radiation very small. Thus, the coupler can be divided into segmented stages as shown in Fig. 2, so that in each stage, the spacing can be regarded as constant.

Optical beam propagation in the segmented stages can be expressed by the mode expansion. Cascading these segmented stages, the propagation characteristics of the whole coupler can be obtained. 


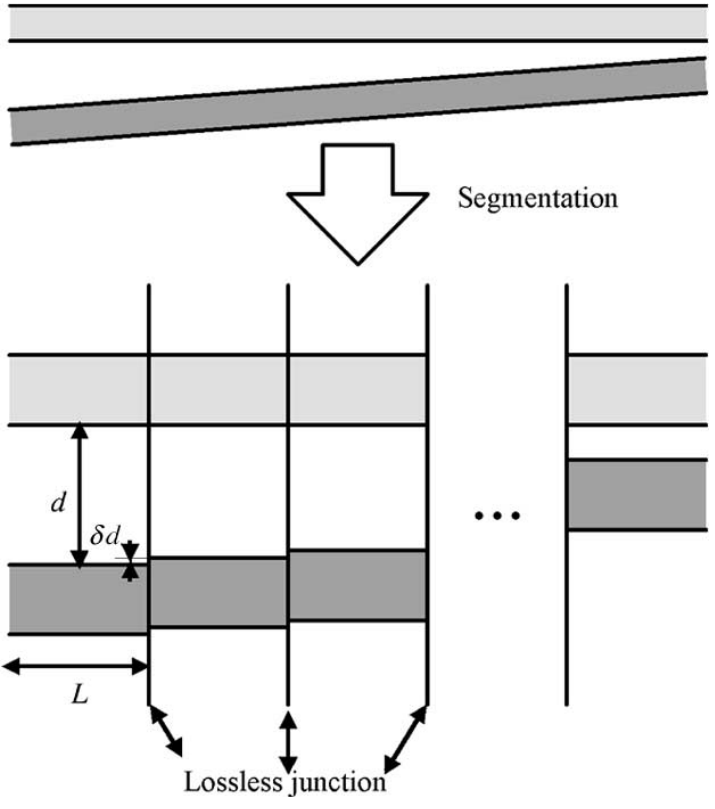

Fig. 2. Modal expansion of eigenmodes in terms of isolated local normal modes.

In this section, the mode expansion in the coupler with constant spacing and the segmentation of the coupler are discussed.

\section{A. Mode Expansion}

Eigenmodes $E_{e}$ and $E_{o}$ in a parallel directional coupler are approximately expressed by the linear combination of isolated local normal modes $E_{1}$ and $E_{2}$ in the individual waveguides as shown in Fig. 2. The relation is given by the following matrix formalism:

$$
\begin{aligned}
{\left[\begin{array}{c}
E_{e} \\
E_{o}
\end{array}\right] } & =\mathbf{C}\left[\begin{array}{l}
E_{1} \\
E_{2}
\end{array}\right] \\
\mathbf{C} & =\left[\begin{array}{ll}
C_{e 1} & C_{e 2} \\
C_{o 1} & C_{o 2}
\end{array}\right]
\end{aligned}
$$

where $C_{i j}$ is the expansion coefficient between the $i$ th coupled eigenmode and the $j$ th isolated local normal mode, and $\mathbf{C}$ is the expansion coefficient matrix.

The incident light amplitudes $E_{1}^{(\mathrm{in})}$ and $E_{2}^{(\mathrm{in})}$ from isolated waveguides are expanded into two coupled eigenmodes $E_{e}$ and $E_{o}$. The coupled eigenmodes propagate with their own propagation constants. Then the superposed field of coupled eigenmodes at the end of the directional coupler is expanded into isolated local normal modes $E_{1}^{\text {(out) }}$ and $E_{2}^{\text {(out) }}$ of the output waveguides. This procedure is expressed in terms of matrix formalism as

$$
\begin{aligned}
{\left[\begin{array}{l}
E_{1}^{(\text {out })} \\
E_{2}^{\text {(out) }}
\end{array}\right] } & =\mathbf{C}^{\dagger} \mathbf{D C}\left[\begin{array}{l}
E_{1}^{(\text {in })} \\
E_{2}^{(\text {in })}
\end{array}\right] \\
\mathbf{D} & =\left[\begin{array}{cc}
\exp \left(-j \beta_{e} L\right) & 0 \\
0 & \exp \left(-j \beta_{o} L\right)
\end{array}\right] .
\end{aligned}
$$

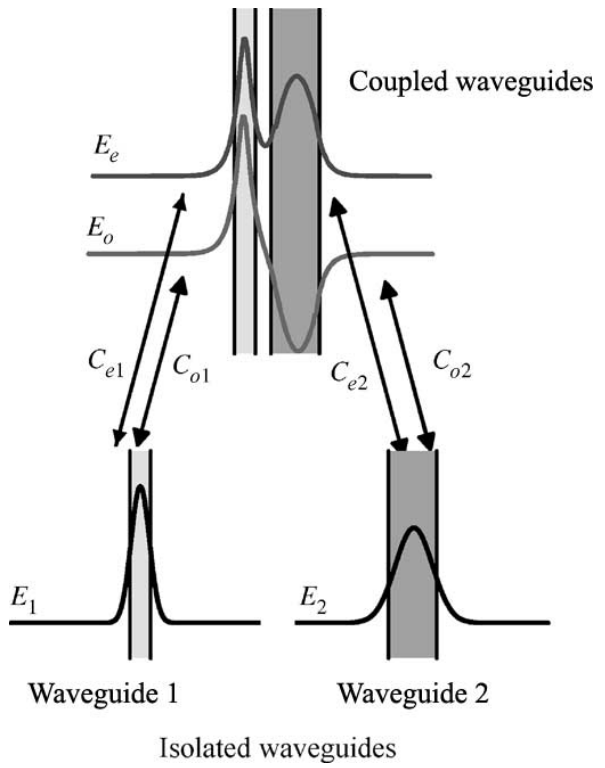

Fig. 3. Segmentation of bent coupled waveguides.

$\mathbf{T}=\mathbf{C}^{\dagger} \mathbf{D C}$ represents the propagation in a segmented parallel directional coupler with segmented coupling length $L$ and a constant spacing $d$.

\section{B. Segmentation}

In the case of bent coupled waveguides, the spacing and the coupling efficiency vary along the propagation distance. Thus, the bent coupled waveguides were modeled by cascading the segmented parallel coupled waveguides [8], [9] as shown in Fig. 3, and the coupling matrix $\mathbf{T}^{(k)}$ at the $k$ th segmented region was calculated. Multiplying these matrices, the coupling matrix of the whole directional coupler $\mathbf{T}_{\text {total }}$ can be obtained. Thus, the output field is expressed by

$$
\begin{aligned}
\mathbf{E}^{(N)} & =\mathbf{T}^{(N-1)} \mathbf{T}^{(N-2)} \cdots \mathbf{T}^{(1)} \mathbf{T}^{(0)} \mathbf{E}^{(0)} \\
\mathbf{T}_{\text {total }} & =\prod_{k=0}^{N} \mathbf{T}^{(k)} .
\end{aligned}
$$

In this analysis, the offset of the optical axis occurs at each connection of segmentations. However, since this modeling is based on the bent coupled waveguide, the shape of which changes smoothly along the propagation axis, the connection loss at the interface between adjacent segmented regions can be ignored. Then in the lossless case, each matrix $\mathbf{T}^{(k)}$ is unitary.

\section{LATERAl ARRANGEMENT OF COUPLER}

Let the coupling efficiency be defined by the ratio of the power of unity input to the output power from the other waveguide of the coupler. Coupling efficiency depends on the spacing between the waveguide centers of the two waveguides, the field profiles of individual waveguides, and the length of the coupling region. 


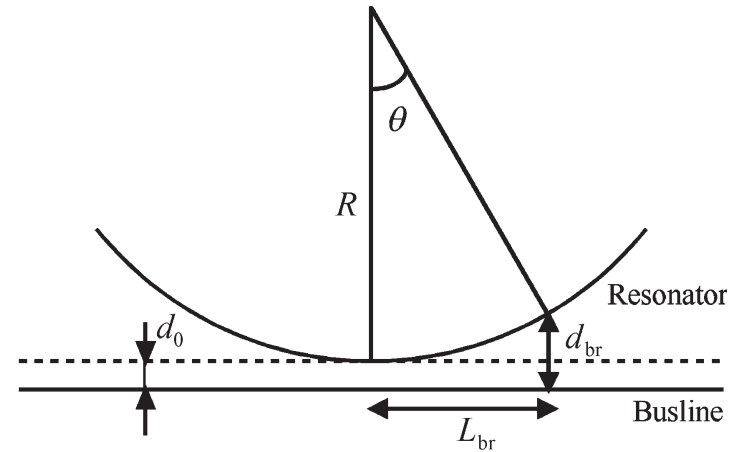

Fig. 4. Lateral structure of coupling region (busline-resonator).

When the waveguides are fabricated in a single layer, the propagation axis of the waveguide lies in the same layer. Then the spacing between the waveguides is given by only the horizontal distance between the waveguides. When the waveguides are arranged in the stacked layer, the vertical distance should be taken into account by using the Pythagorean theorem.

\section{A. Coupling Between Busline and Resonator}

Fig. 4 shows the coupling region between the busline and the ring resonator. Although the light propagates the busline waveguide and the ring resonator waveguide in different directions, the coupling occurs almost in the limited area where the two waveguides can be regarded as a parallel coupled waveguide with close spacing. The waveguide spacing $d_{\mathrm{br}}$ is approximately expressed by

$$
d_{\mathrm{br}} \simeq \frac{1}{2} R \theta^{2}+d_{0}
$$

where $R$ is the radius of the ring resonator, $\theta$ is the azimuthal angle defined as shown in Fig. 4, and $d_{0}$ is the nearest distance between waveguide centers. When the waveguides are crossing each other, $d_{0}<0$.

The propagation distance from the point with the minimum spacing is given by $L \simeq R \theta$. Thus, $L$ is expressed as a function of $d_{\mathrm{br}}$ in terms of (7) as

$$
L_{\mathrm{br}} \simeq \sqrt{2 R\left(d_{\mathrm{br}}-d_{0}\right)} .
$$

If the coupling region is divided into segmented parallel coupled waveguides with equal propagation distance, then the spacing of segmented coupled waveguides increases parabolically with the increase of $L$, and the discontinuity between segmented regions increases. Therefore, the length of the segmented coupled region should be determined so as to keep the discontinuity constant. Then the local propagation distance in each segmented coupled region should be determined by

$$
l_{\mathrm{br}}\left(d_{\mathrm{br}}\right)=\delta d \sqrt{\frac{R}{2\left(d_{\mathrm{br}}-d 0\right)}}
$$

where $\delta d$ is the segment step, which is the lateral step of digitized location of sore boundary.

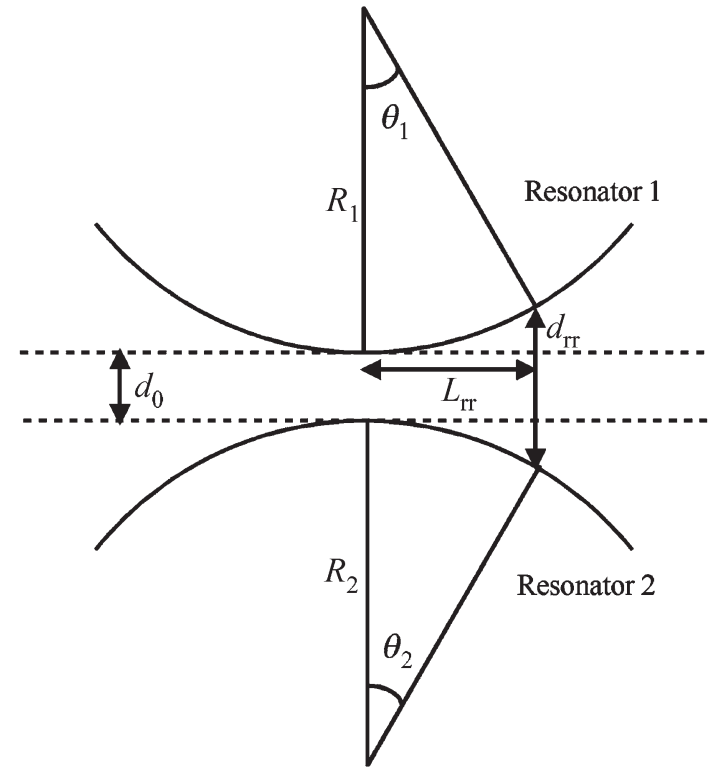

Fig. 5. Lateral structure of coupling region (resonator-resonator).

\section{B. Coupling Between Resonators}

Fig. 5 shows the coupling region between two resonators. The waveguide spacing $d_{\mathrm{rr}}$ is given by

$$
d_{\mathrm{rr}} \simeq \frac{1}{2} R_{1} \theta_{1}^{2}+\frac{1}{2} R_{2} \theta_{2}^{2}+d_{0}
$$

where $R_{1}$ and $R_{2}$ are the radii of two ring resonators, respectively.

The propagation distance from the point with the minimum spacing is given by $L \simeq R_{1} \theta_{1}=R_{2} \theta_{2}$. Then $L$ is expressed as a function of $d_{\text {rr }}$ as

$$
L_{\mathrm{rr}} \simeq \sqrt{2 \frac{R_{1} R_{2}}{R_{1}+R_{2}}\left(d_{\mathrm{rr}}-d_{0}\right)} .
$$

Then the local propagation distance corresponding to the constant step of the spacing at the segmented region is given by

$$
l_{\mathrm{rr}}\left(d_{\mathrm{rr}}\right)=\delta d \sqrt{\frac{R_{1} R_{2}}{2\left(R_{1}+R_{2}\right)\left(d_{\mathrm{rr}}-d_{0}\right)}} .
$$

When $R_{1}=R_{2}$, then $\sqrt{2} l_{\mathrm{rr}}=l_{\mathrm{br}}$. Thus, the coupling between the resonators is about half of the coupling between the busline and resonator.

\section{Light Propagation in Two-Dimensional Directional COUPLER}

The light propagation in a coupled structure with unit power input was calculated as shown in Fig. 6. The power transfer of the busline waveguide was calculated by the present method and the two-dimensional (2-D) FDTD simulation, and the result is shown in Fig. 7. The mesh size of the FDTD simulation and the segment step $\delta d$ of the present method were both $0.05 \mu \mathrm{m}$. Computational domains are threefold larger than the core cross-sectional size in the mode solver calculation, and 


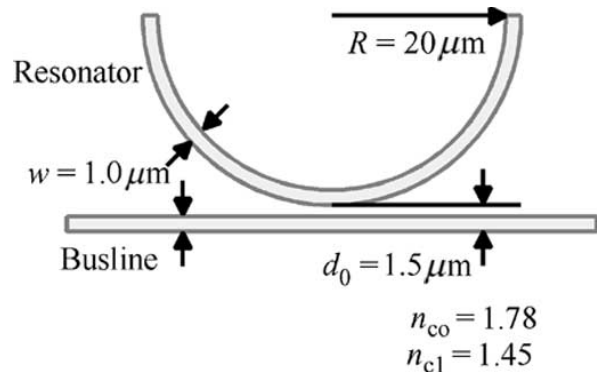

Fig. 6. Directional coupler consisting of straight and bent waveguides.

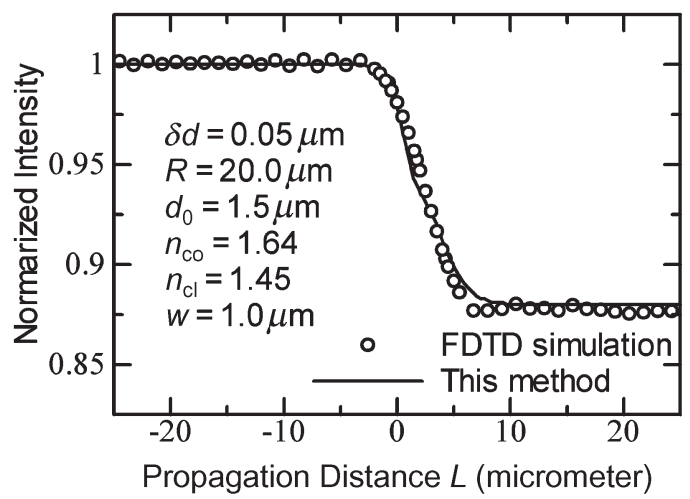

Fig. 7. Power transfer in directional coupler with straight and bent waveguides.

fivefold larger than the core width in the FDTD simulation. The perfectly matched layer (PML) was used as a boundary condition. The local normal mode of bent waveguide was calculated by gradient index approximation. The field profiles $E_{e}$ and $E_{o}$ of each segmented waveguide and $E_{1}$ and $E_{2}$ of isolated waveguides, which are required to determine the expansion coefficient matrix given in (2), were calculated by a finite-difference mode solver [Apollo Photonics Solution Suite (APSS) by Apollo Photonics Inc.]. The 2-D and 3-D FDTD tools included in the same software (APSS) were also used.

It can be seen in this figure that the calculated result of the present method almost agrees with that of the FDTD simulation.

Fig. 8 shows the transmittance of the busline waveguide when the segment step $\delta d$ was changed. As the segment step was decreased, the transmittance converges to a constant as in the case with the FDTD simulation.

This analysis method can reduce the computation time by more than one order of magnitude compared with the FDTD simulation, because the expansion coefficient matrix can be repeatedly used when the same cross-sectional structure appears several times as in the case of Fig. 6.

\section{Light Propagation in 3-D Directional Coupler}

The light propagation in a 3-D coupled structure with unit power input by the present method and the 3-D FDTD simulation was calculated, and results are shown in Fig. 9. It can be seen in this figure that the calculated result of the present method almost agrees with that of the FDTD simulation. The mesh size of FDTD was $40 \mathrm{~nm}$, and the segment step of this

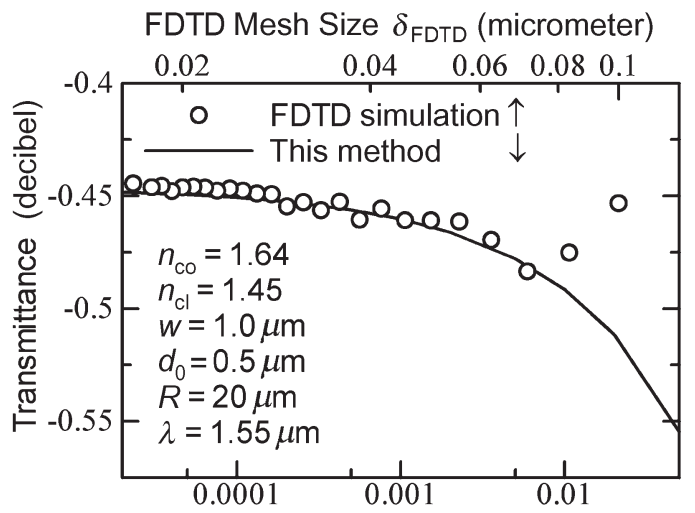

Segment Step in This Analysis $\delta d$ (micrometer)

Fig. 8. Error of calculation.

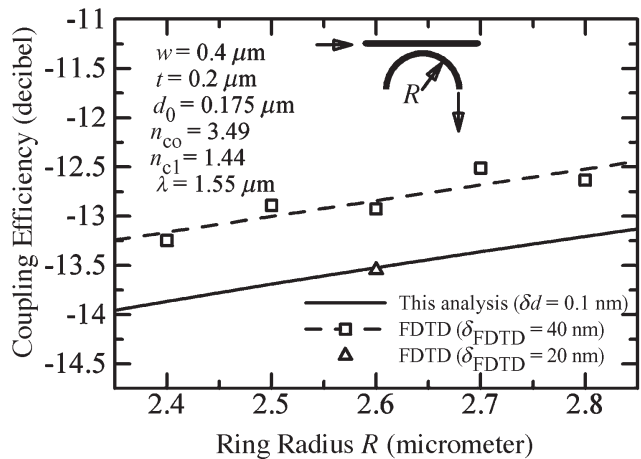

Fig. 9. Coupling efficiency (single layer, 3-D).

analysis was $1 \mathrm{~nm}$. The coupling efficiency calculated by the 3-D FDTD simulation was larger than that calculated by this analysis. This is because the mesh size of the FDTD simulation was not small enough to obtain an accurate coupling efficiency. The computation time of the present method to obtain one point in Fig. 9 was about 10 min, including the calculation of modal fields by the mode solver and the calculation of matrices. On the other hand, it took more than several hours to calculate one point in Fig. 9 by the 3-D FDTD simulation. Therefore, it is not so difficult to calculate the coupling efficiency for many different conditions by this method. This is the reason why the calculated result of this method in Fig. 9 is shown by a solid curve while that of the 3-D FDTD analysis is shown by only several points.

\section{Light PRopagation of Stacked DiRECTIONAL COUPLER}

Next, suppose the coupling between the waveguides is arranged in different layers as shown in Fig. 11. A 3-D FDTD simulation can be used. However, 3-D FDTD simulation can be applied only to a small area due to the limitation of memory and time. In this analysis, the required parameters are the expansion coefficients, the propagation constants, and the segmented coupling lengths $L$ in which the busline waveguide and the resonator waveguide were considered to be separated by a constant spacing $\left(d_{0}, t_{s}\right)$, as shown in Fig. 11. The expansion coefficient and the propagation constant were obtained from the 


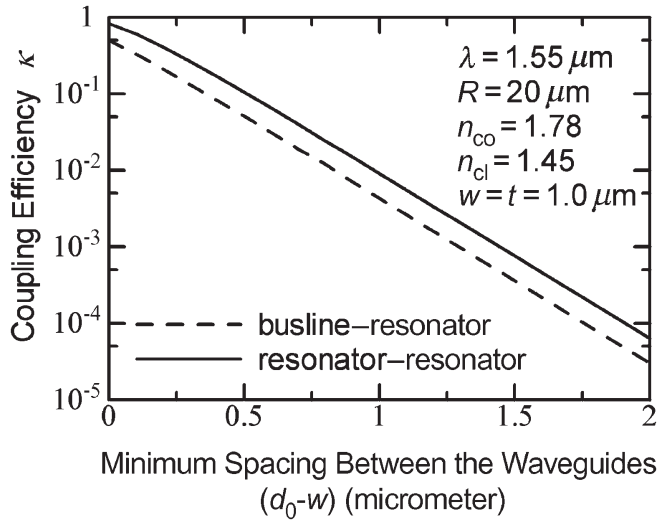

Fig. 10. Coupling efficiency (single layer).

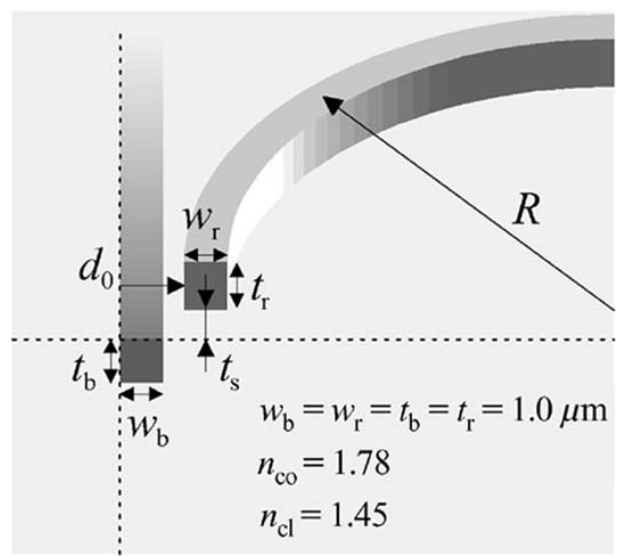

Fig. 11. 3-D arrangement of coupling region.

coupled-mode field in each cross-sectional structure, and the segmented coupling lengths were calculated from the geometrical arrangement in the same way as described in Section III. Then, the coupling efficiency between the busline waveguide and the resonator waveguide in different layers can be obtained.

\section{A. Coupling Between Busline and Resonator}

Fig. 10 shows the power coupling efficiency in the case that the waveguides are arranged in the same layer (dashed line). The coupling efficiency decreases exponentially with increased minimum spacing.

Next, the coupling efficiency between the straight busline waveguide and the bent resonator waveguide $(R=20 \mu \mathrm{m})$, which are arranged as shown in Fig. 11, was calculated using this new method. The calculated result is shown in Fig. 12 . The strongest coupling is obtained around $d_{0} \simeq-0.5 \mu \mathrm{m}$. The coupling efficiency also decreases exponentionally with increased spacing both in the lateral and vertical directions. When $d_{0}=0$, the length of the segmented coupling region with minimum spacing is the longest compared with other cases with $d_{0} \neq 0$. However, when $d_{0} \simeq-0.5 \mu \mathrm{m}$ rather than $d_{0}=0 \mu \mathrm{m}$, the total efficiency is strong. This seems to be caused by the fact that the accumulation of coupled power along the propagation axis for $d_{0} \simeq-0.5 \mu \mathrm{m}$ is stronger than that for $d_{0}=0 \mu \mathrm{m}$, because the effective coupler length for $d_{0}=-0.5 \mu \mathrm{m}$ is longer than that for $d_{0}=0 \mu \mathrm{m}$. In addition,

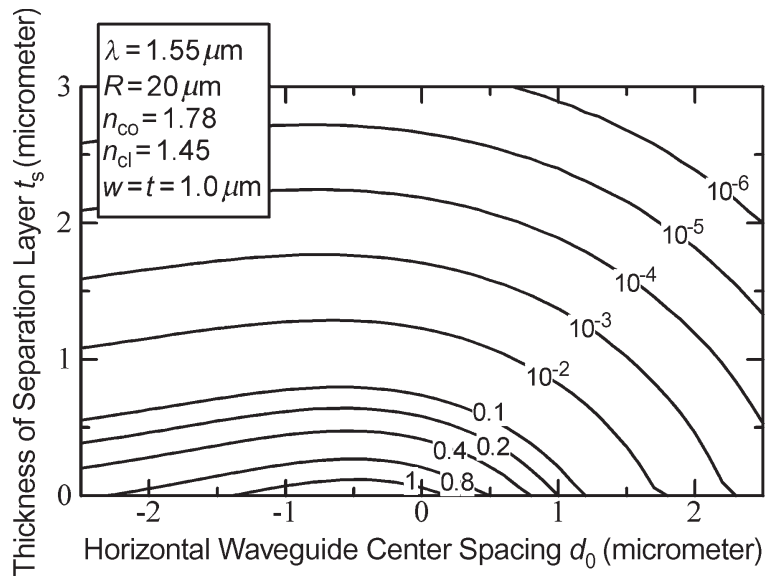

Fig. 12. Coupling efficiency (busline-resonator).

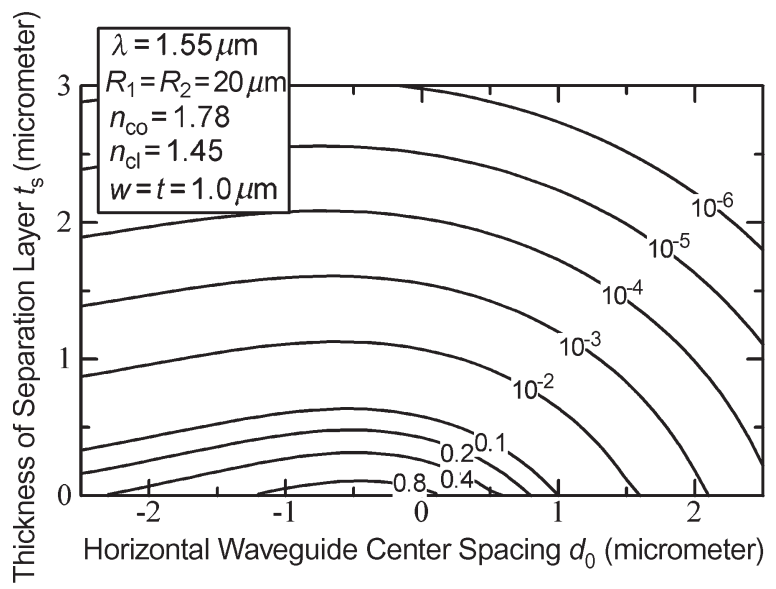

Fig. 13. Coupling efficiency (resonator-resonator).

at any thickness of separation layer, the coupling efficiency is almost constant around $d_{0} \simeq-0.5 \mu \mathrm{m}$. This means that the fabrication tolerance for the lateral misalignment is large, and this fact is the advantage of vertical coupling.

\section{B. Coupling Between Resonators}

The power coupling efficiency between resonators is also shown by the solid line in Fig. 10 when the waveguides are arranged in the same layer. The coupling efficiency has almost the same dependence on the lateral spacing as in the coupling between busline and resonator. However, the coupling efficiency is about half of that between the busline and resonator.

Fig. 13 shows the coupling efficiency between the resonator waveguides $\left(R_{1}=R_{2}=20 \mu \mathrm{m}\right)$ as a function of horizontal and vertical spacings when they are arranged in different layers. The dependence of coupling efficiency on the horizontal and vertical spacings is almost similar to that between the busline and the resonator. However, the coupling efficiency is about half of that between the busline and the resonator.

\section{Comparison of Single-Layer and Stacked Configuration}

Fig. 14 shows the calculated power coupling efficiency against the ring radius for the cases of lateral waveguide spacing 


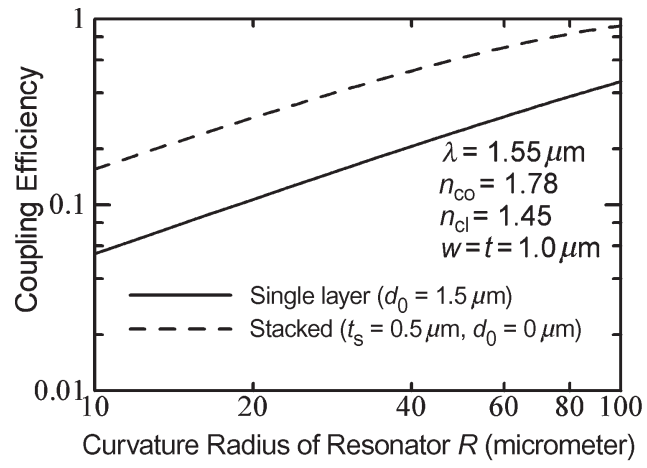

Fig. 14. Coupling efficiency versus ring radius for single layer and stacked couplers.

of $0.5 \mu \mathrm{m}$ in the lateral coupling (single layer) and lateral waveguide center spacing of $0 \mu \mathrm{m}$ with $0.5-\mu \mathrm{m}$ separation layer in the vertical coupling. Even if the single-layer configuration and the stacked configuration have the same minimum spacing of $0.5 \mu \mathrm{m}$ between waveguides, the stacked configuration can achieve stronger coupling than the single-layer configuration by more than threefold. This is because the stacked configuration has longer coupling region than the single-layer configuration.

In the microring resonator filters, a coupling efficiency of $5 \%-10 \%$ is required to obtain an appropriate value of bandwidth suitable for WDM transmission. For example, free spectrum range (FSR) is $20 \mathrm{~nm}$ for the ring radius of $10 \mu \mathrm{m}$ and $n_{\text {eff }}=1.9$. When the bandwidth of $0.4 \mathrm{~nm}(50 \mathrm{GHz}$ at $\lambda=1550 \mathrm{~nm}$ ) is required, the coupling efficiency of $6 \%$ is needed to give a finesse of 50 . It is possible to achieve this coupling efficiency using the stacked configuration, but it is not so easy using the single-layer coupler as seen in Fig. 14. This is because the vertical spacing can be precisely controlled by the thickness control of the separation layer, which is much easier than the gap control by etching in the case of lateral (single layer) coupling. Therefore, the stacked configuration is much more effective for the microring resonator.

\section{CONCLUSION}

A novel fast analysis method of three-dimensional (3-D) coupled waveguides using the segmentation of coupler and local normal mode has been proposed and demonstrated.

In this analysis, it is not necessary to calculate all the crosssectional modal fields, because interpolation can be used to increase the resolution of waveguide spacing. When the crosssectional structure appears several times in the propagation direction as is the case of coupling between bent and straight waveguides, this method does not need to calculate the modal field again. In this analysis, not all of the bending effects were considered. However, most of the coupling occurs in the region where the waveguides are assumed to be parallel. Therefore, this analysis can analyze effectively the 3-D coupled structure.

\section{REFERENCES}

[1] S. Suzuki, K. Shuto, and Y. Hibino, "Integrated optic ring resonators with two stacked layers of silica waveguides on Si," IEEE Photon. Technol. Lett., vol. 4, no. 11, pp. 1256-1258, Nov. 1992.

[2] B. E. Little, S. T. Chu, J. Forsi, G. Steinmeyer, E. Thoen, H. A. Haus, E. P. Ippen, L. Kimerling, and W. Grrene, "Microresonators for integrated optical devices," Optics Photon. News, vol. 9, no. 12, pp. 32-33, 1998.

[3] C. K. Madsen, "Efficient architectures for exactly realizing optical filters with optimum bandpass designs," IEEE Photon. Technol. Lett., vol. 10, no. 8, pp. 1136-1138, Aug. 1998.

[4] S. T. Chu, B. E. Little, W. Pan, T. Kaneko, S. Sato, and Y. Kokubun, "An eight-channel add-drop filter using vertically coupled microring resonators over a cross grid," IEEE Photon. Technol. Lett., vol. 11, no. 6, pp. 691-693, Jun. 1999.

[5] S. T. Chu, B. E. Little, W. Pan, T. Kaneko, and Y. Kokubun, "Second-order filter response from parallel coupled glass microring resonators," IEEE Photon. Technol. Lett., vol. 11, no. 11, pp. 1423-1425, Nov. 1999.

[6] B. E. Little, S. T. Chu, W. Pan, and Y. Kokubun, "Microring resonator arrays for VLSI photonics," IEEE Photon. Technol. Lett., vol. 12, no. 3, pp. 323-325, Mar. 2000.

[7] C. K. Madsen and J. H. Zhao, "A general planar waveguide autoregressive optical filter," J. Lightw. Technol., vol. 14, no. 3, pp. 437-447, Mar. 1996.

[8] R. N. Thurston, E. Kapon, and Y. Silberberg, "Analysis of mode separation in multichannel branching waveguides," IEEE J. Quantum Electron., vol. 23, no. 8, pp. 1245-1255, Aug. 1987.

[9] H. A. Haus and W. P. Huang, "Mode coupling in tapered structures," J. Lightw. Technol., vol. 7, no. 4, pp. 729-730, Apr. 1989.

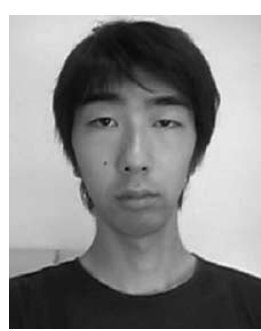

Tomoyuki Kato was born in Saitama, Japan, on January 3, 1979. He received the B.E. and M.E degrees in electrical and computer engineering from Yokohama National University, Yokohama, Japan, in 2001 and 2003, respectively.

His main interests include optical waveguide-type functional devices.

Mr. Kato is a Member of the Institute of Electronics, Information and Communication Engineers (IEICE) of Japan.

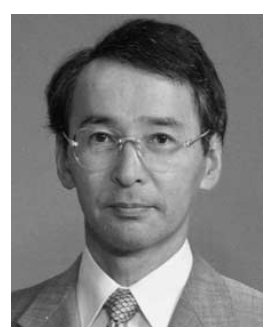

Yasuo Kokubun (M'03) was born in Fukushima, Japan, on July 7, 1952. He received the B.E. degree from Yokohama National University, Yokohama, Japan, in 1975, and the M.E. and Dr. Eng. degrees from Tokyo Institute of Technology, Tokyo, Japan, in 1977 and 1980, respectively.

After he worked for the Research Laboratory of Precision Machinery and Electronics, Tokyo Institute of Technology, as a Research Associate from 1980 to 1983, he joined the Yokohama National University as an Associate Professor, and is now a Professor of the Department of Electrical and Computer Engineering. His current research is in integrated photonics, especially waveguide-type functional devices and threedimensional (3-D) integrated photonics. From 1984 to 1985, he was with AT\&T Bell Laboratories, Holmdel, NJ, as a Visiting Researcher and was engaged in a novel waveguide on semiconductor substrate antiresonant reflecting optical waveguide (ARROW) for integrated optics. From 1996 to 1999, he served as the project leader of the "Three-Dimensional Microphotonics Project at the Kanagawa Academy of Science and Technology."

Prof. Kokubun is a Member of the Japan Society of Applied Physics and the Optical Society of America (OSA). 